\title{
Gain-of-function $\beta$-catenin in the uterine mesenchyme leads to impaired implantation and decidualization
}

\author{
Amanda L Patterson, Jamieson Pirochta, Stephanie Y Tufano and Jose M Teixeira \\ Department of Obstetrics, Gynecology, and Reproductive Biology, College of Human Medicine, \\ Michigan State University, Grand Rapids, Michigan, USA
}

Correspondence should be addressed to $J \mathrm{M}$ Teixeira Email

jose.teixeira@hc.msu.edu

\begin{abstract}
Embryo implantation and endometrial decidualization are critical events that occur during early pregnancy in humans and mice, and perturbation in either can result in infertility. WNT signaling through the canonical $\beta$-catenin pathway plays a pivotal role in embryonic Müllerian duct development, postnatal uterine maturation and establishment of pregnancy. Loss of $\beta$-catenin in the Müllerian duct mesenchyme (MDM)-derived stroma and myometrium results in impaired decidualization and infertility, whereas gain-of-function (GOF) results in the formation of mesenchymal tumors and subfertility attributed to malformed oviducts. We hypothesized that GOF $\beta$-catenin further contributes to sub-fertility through improper stromal and epithelial cell signaling during embryo implantation and decidualization. We show that mice with GOF $\beta$-catenin in MDM-derived stroma and myometrium have reduced implantation sites after embryo transfer and decreased decidualization. On day 4.5 of pseudopregnancy or in mice treated with progesterone and estrogen to mimic early pregnancy, the estrogen-LIF-ERK and progesterone-IHH pathways remain predominantly intact in GOF $\beta$-catenin mice; however, JAK/STAT signaling is altered. pSTAT3 is significantly reduced in GOF $\beta$-catenin mice and expression of downstream epithelial junctional complex factors, Ctnna1 and Cldn1, is increased. We also show that purified stromal cells from GOF $\beta$-catenin uteri, when removed from epithelial cell influence and provided with the appropriate hormonal stimuli, are able to decidualize in vitro indicating that the cells are intrinsically capable of decidualization. Taken together, these results suggest that dysregulated $\beta$-catenin activity in the stroma affects epithelial cell STAT3 signaling and ultimately embryo implantation and stromal decidualization.
\end{abstract}

\section{Key Words}

- $\beta$-catenin

- embryo implantation

- decidualization

- stromal-epithelial crosstalk

- STAT3
Journal of Endocrinology (2017) 233, 119-130

\section{Introduction}

Two of the most crucial events in early pregnancy in species with invasively implanting embryos, such as humans and mice, are embryo implantation and endometrial decidualization. Embryo implantation is a tightly regulated series of events, beginning with apposition of the embryo to the uterine luminal epithelium, followed by physical attachment to the epithelium and culminating with penetration beyond the basement membrane into the underlying stroma (Wang \& Dey 2006). Decidualization, which is initiated during embryo attachment in concert 
with proper hormone priming, is the process by which endometrial stromal cells terminally differentiate into decidual cells to form the decidua. The decidua is required for the establishment and maintenance of early pregnancy as it provides a source of growth factors and cytokines that support embryo development, modulates maternal immune responses, regulates embryo invasion and supports maternal angiogenesis (Wang \& Dey 2006, Ramathal et al. 2010). Perturbation at any stage of implantation or decidualization will normally result in early pregnancy loss.

The window of uterine receptivity is a tightly regulated period in which the endometrium is receptive to embryo implantation. Priming of the uterus to become receptive is initiated by ovarian-derived estrogen and progesterone and requires intricately coordinated signaling between the luminal and glandular epithelia (LE and GE, respectively) and the stroma (Wang \& Dey 2006, Hantak et al. 2014). Proper hormone signaling and stromal-epithelial crosstalk is also necessary for decidualization. For example, one of the most well-studied stromal-epithelial crosstalk pathways involved in decidualization is the Indian hedgehog (IHH) pathway (Pawar et al. 2014). In brief, progesterone signals through the progesterone receptor (PR) in the LE resulting in the expression of IHH that then binds its receptor, patched1 (PTCH1) in the stroma (Matsumoto et al. 2002, Takamoto et al. 2002, Lee et al. 2006, Rubel et al. 2012). This initiates a downstream signaling cascade in the stroma that involves the expression of chicken ovalbumin upstream promoter-transcription factor II (COUP-TFII), bone morphogenetic protein 2 (BMP2) and wingless-type MMTV integration site family 4 (WNT4) and leads to decidualization (Lee et al. 2006, 2007, Kurihara et al. 2007, Li et al. 2007, 2013, Franco et al. 2011). Loss in function of any of the previously mentioned factors results in impaired decidualization and subsequent infertility.

It is known that members of the WNT family of glycoproteins play pivotal roles in embryonic Müllerian duct development, postnatal uterine maturation and establishment of early pregnancy (Parr \& McMahon 1998, Vainio et al. 1999, Mericskay et al. 2004, Dunlap et al. 2011, Franco et al. 2011, Prunskaite-Hyyrylainen et al. 2016). $\beta$-Catenin, which is the downstream effector of canonical WNT signaling, is an evolutionarily conserved protein with two major functions: (1) it can bind to cadherin proteins at the plasma membrane and contribute to formation of adherens junctions and (2) upon WNT stimulation, it can regulate the transcription of target genes through interaction with the TCF/LEF family of transcription factors (Valenta et al. 2012). In the absence of WNT signaling, $\beta$-catenin is located in the cytosol, bound by a destruction complex consisting of APC, AXIN1, GSK3 $\beta$ and CK1. GSK3 $\beta$ and CK1 phosphorylate $\beta$-catenin on serines 33,37 and 45 and threonine 41 , which signals for ubiquitination and proteosomal degradation. Similar to WNT proteins, $\beta$-catenin has been shown through mouse models to be required for proper uterine development and function (Arango et al. 2005, Mohamed et al. 2005, Deutscher \& Hung-Chang Yao 2007, Zhang et al. 2012). Our lab and others have shown that loss of $\beta$-catenin in Müllerian duct mesenchyme (MDM)-derived tissues (stroma and myometrium) results in a myocyte to adipocyte conversion in the myometrium and shallow stroma (Arango et al. 2005) as well as complete inhibition of decidualization (Zhang et al. 2012). Conversely, mice that express a mutated gain-of-function (GOF) form of $\beta$-catenin in the MDM have a reduced number of glands (Stewart et al. 2013), develop myometrial and stromal tumors (Tanwar et al. 2009) and are sub-fertile (Boerboom et al. 2005). This sub-fertility has been primarily attributed to malformed oviducts that impede oocyte, sperm and embryo transport (Stewart et al. 2013). However, the impact of GOF $\beta$-catenin on uterine function in the context of early pregnancy has not been reported. We therefore sought to gain more insight into the role of stromal $\beta$-catenin in implantation and decidualization using mice with GOF $\beta$-catenin in the uterine MDM. We hypothesized that the sub-fertility phenotype of GOF $\beta$-catenin mice is due, in part, to improper stromal and epithelial cell signaling during embryo implantation and decidualization.

\section{Materials and methods}

\section{Animals}

Animal protocols were approved by the Michigan State University Institutional Animal Care and Use Committee. To generate mice with GOF $\beta$-catenin in MDM-derived stroma and myometrium, mice with anti-Müllerian hormone type II receptor (Amhr2) promoter-driven Cre recombinase $\left(A m h r 2^{C r e /}\right)$, kindly provided by Dr. Richard Behringer (Jamin et al. 2002), were mated to $\beta$-catenin (Ctnnb1) exon 3 floxed (Ctnnb1 lox(ex3)/lox(ex3)) mice, kindly provided by Dr. Makoto Taketo (Harada et al. 1999). The resulting offspring were either controls (Amhr2Cre/+ or Ctnnb $1^{\text {lox(ex3)/+) }}$ or Amhr2 $2^{\mathrm{Cre} /++}$; Ctnnb $1^{\text {lox }(e x 3) /++}$ (GOF $\beta$-catenin)mutant mice in which exon 3 of Ctnnb1 was deleted, 
which resulted in a stabilized (e.g. non-degradable) form of $\beta$-catenin. Serum hormone measurements for progesterone and estradiol were performed by the University of Virginia Ligand Core. Sample sizes are indicated in figure legends.

\section{Embryo transfer}

Day 2.5 embryos were purchased from Jackson Labs and after 24-h culture, day 3.5 embryos were surgically transferred into control and GOF $\beta$-catenin surrogate mice on day of pseudopregnancy (DOPP) 2.5. Surrogates were prepared by placing sexually mature (3- to 4-month old) females with vasectomized males and designating DOPP 0.5 on the morning a vaginal plug was observed. To visualize implanted embryos on day 5.5 of pregnancy, mice were injected with $100 \mu \mathrm{L}$ Chicago Blue dye in the tail vein and were killed 3-5 min later. The number of embryos implanted as a percentage of the number transferred into each uterine horn per mouse was calculated and statistics were performed using the unpaired $T$-test.

\section{Pseudopregnancy and induced decidualization}

Pseudopregnancy was induced by placing sexually mature (3- to 4-month old) control and GOF $\beta$-catenin female mice with vasectomized CD1 males. For DOPP 4.5 tissue collections, females were killed and uteri were processed for protein isolation and paraffin embedding. To induce decidualization, $20 \mu \mathrm{L}$ of sesame oil was injected into the uterine lumen on DOPP 4. On DOPP 9, mice were killed and uterine wet weights were obtained for the unstimulated (control) and stimulated horns for each mouse. Statistical analysis was performed by comparing the increase in weight of the stimulated horn to the unstimulated horn by 2-way ANOVA.

\section{Early pregnancy hormone stimulation and induced decidualization}

To mimic hormone stimulation of uteri with exogenous estradiol $\left(\mathrm{E}_{2}\right)$ and progesterone $\left(\mathrm{P}_{4}\right)$, female mice were first ovariectomized and allowed to clear endogenous hormones for one week. Mice then received priming injections of $\mathrm{E}_{2}$ (Sigma; 100 ng in $100 \mu \mathrm{L}$ sesame oil, s.c.) for two days, no hormones for two days, and then followed by four daily injections of $\mathrm{E}_{2}+\mathrm{P}_{4}$ (Sigma; $10 \mathrm{ng}$ $\mathrm{E}_{2}+1 \mathrm{mg} \mathrm{P}_{4}$ in $150 \mu \mathrm{L}$ sesame oil, s.c.). Six hours after the last injection on the fourth day, mice were killed and uterine horns were collected and processed for protein and RNA isolation and paraffin embedding. For induced decidualization, after estrogen priming, mice were implanted with a progesterone pellet $(1 \mathrm{mg}$ per day release rate; Innovative Research of America, Sarasota, FL, USA) and given daily $\mathrm{E}_{2}$ injections as described previously. Six hours after the third $\mathrm{E}_{2}$ injection, one uterine horn was scratched to induce decidualization, and uteri were collected 5 days later.

\section{RNA isolation, cDNA synthesis and qRT-PCR}

Total RNA was extracted from whole uteri or from stromal cells that underwent decidualization in vitro using the RNeasy Mini Kit (Qiagen) per manufacturer's instructions, including DNase I treatment. RNA was reverse transcribed using SuperScript IV Reverse Transcriptase (Invitrogen) with oligo-dT primers. Quantitative real-time PCR (qRT-PCR) was then performed for genes of interest with primer sets $(0.2 \mu \mathrm{M}$ final concentration each) listed in Supplementary Table 1 (see section on supplementary data given at the end of this article) using Power SYBR Green Master Mix (Applied Biosystems) by a ViiA 7 Real-Time PCR System (Applied Biosystems). Rpl19 and Gapdh mRNA were used as internal controls and a mock reverse transcriptase was also included as a template to confirm the absence of genomic DNA. Relative quantification of mRNA was determined in which ratios for each gene were established using Rpl19 or Gapdh as a reference.

\section{Immunohistochemistry}

Uteri were fixed in $4 \%$ paraformaldehyde and embedded in paraffin wax. $6 \mu \mathrm{m}$ thick sections were deparaffinized in xylene and rehydrated. Antigen retrieval was done in sodium citrate buffer ( $\mathrm{pH}$ 6.0) and sections were processed for IHC using the EnVision+ System-HRP Kit (Dako) according to manufacturer's instructions. Briefly, the sections were washed with Tris buffered saline with $0.1 \%$ Tween20 (TBST), incubated in peroxidase block, washed in TBST and then incubated with primary antibody diluted in $1 \%$ bovine serum albumin in phosphate buffered saline (PBS) at the indicated dilutions in Supplementary Table 2 overnight at $4^{\circ} \mathrm{C}$. The sections were then washed with TBST, incubated for $30 \mathrm{~min}$ at room temperature (RT) with anti-rabbit-HRP-conjugated polymer, and then incubated with DAB (3,3'-diaminobenzidine) substrate. Sections were counterstained and visualized. Photos were taken with a Nikon Eclipse Ni microscope and DS-Fi2 digital camera.

Published by Bioscientifica Ltd 


\section{Western blot}

Protein was isolated using RIPA (radioimmunoprecipitation assay) buffer with a protease and phosphatase inhibitor cocktail (Sigma) from whole uteri. $20 \mu \mathrm{g}$ of protein from each sample was boiled with sodium dodecyl sulfatepolyacrylamide (SDS) sample loading buffer, subjected to electrophoresis, and then transferred to nitrocellulose (Invitrogen) for protein detection. Membranes were blocked using PBS with 5\% non-fat milk for $1 \mathrm{~h}$ at RT and then probed overnight at $4^{\circ} \mathrm{C}$ with antibodies listed in Supplementary Table 2. After incubation for $1 \mathrm{~h}$ at RT with HRP-conjugated donkey anti-mouse or anti-rabbit secondary antibody (1:10,000; Jackson ImmunoResearch), protein bands were visualized by the addition of ECL substrate (GE Healthcare Bio-Sciences) and film was developed and scanned. $\beta$-Tubulin or $\beta$-actin levels were used as loading controls, and the band intensities were calculated using ImageJ software.

\section{Stromal cell isolation and in vitro decidualization}

Four- to six-month-old control and GOF $\beta$-catenin mice were killed and $n=3-4$ uteri were collected and pooled per genotype for $n=3$ and 4 independent experiments, respectively. Uteri were washed in PBS with $1 \%$ antibiotics/antimycotics, cut in half lengthwise to expose the epithelium and then incubated in $0.25 \%$ trypsin-EDTA (Sigma) in PBS for $30 \mathrm{~min}$ at $37^{\circ} \mathrm{C}$ to release epithelial cells, which were discarded. The remaining pieces of tissue were incubated at $37^{\circ} \mathrm{C}$ in DMEM/F12 (Invitrogen) containing $0.5 \mathrm{mg} / \mathrm{mL}$ Collagenase Type II (Sigma) $1 \mathrm{mg} / \mathrm{mL}$ (400units $/ \mathrm{mL}$ ) DNase I (Sigma), $5 \mathrm{mM}$ $\mathrm{MgCl}_{2}$ and $10 \% \mathrm{FBS}$ (Invitrogen) for $1-2 \mathrm{~h}$ to release stromal cells. Cell suspensions were passed through a $40 \mu \mathrm{M}$ cell strainer to remove undigested tissue and the myometrium, and then incubated in ACK Lysing buffer (Invitrogen) for $5 \mathrm{~min}$ at RT. Cells were washed in PBS and then resuspended in fresh media (DMEM/F12 with antibiotics/antimycotics and 10\% FBS) and expanded in culture. After expansion, any remaining epithelial cells from the digestion process were removed using MACS MicroBead Technology (Miltenyi Biotec, San Diego, CA, USA) according to the manufacturer's instructions. Briefly, cells were trypsinized, washed with PBS solution (PBS containing $0.5 \% \mathrm{BSA}$ and $2 \mathrm{mM}$ EDTA) and incubated with anti-mouse EpCam (CD326)-PE antibody for $10 \mathrm{~min}$ at $4^{\circ} \mathrm{C}$. After washing with PBS solution, labeled cells were incubated with anti-PE MicroBeads, and then passed over a MACS MS Column. Purified stromal cells were plated at 40,000 cells per well in 24-well plates and treated with $10 \mathrm{nM}$ estradiol (Sigma) and $1 \mu \mathrm{M}$ progesterone (Sigma) to induce decidualization ( $\mathrm{Li}$ et al. 2007) or remained untreated in phenol red-free DMEM/F12 with 2\% charcoal-stripped FBS. Media was changed every other day, and treatment continued for eight days to induce decidualization. All experiments were performed on stromal cells at passage 3 or earlier, and decidualization was confirmed by mRNA expression of Prl8a2 (Li et al. 2007) normalized to Gapdh.

\section{Results}

\section{Embryo implantation and endometrial decidualization are impaired in GOF $\beta$-catenin-mutant mice}

Previous reports have demonstrated that mice with GOF $\beta$-catenin in the Müllerian duct mesenchyme (MDM; i.e. stroma and myometrium) have malformed oviducts that result in sub-fertility (Stewart etal. 2013). Therefore, to study possible uterine-specific fertility defects, we performed embryo transfer to bypass the malformed oviducts. Embryos from CD1 donors were transferred into one uterine horn of control or GOF $\beta$-catenin pseudopregnant mice. Uteri were collected on day of pregnancy (DOP) 5.5, and implantation sites were visualized using Chicago Blue dye. Uteri from GOF $\beta$-catenin mice had fewer implantation sites compared to those from controls (Fig. 1A). To determine if reduced embryo implantation was due to a decidualization defect, uterine wet weights were measured on day of pseudopregnancy (DOPP) 9. Although control mice had an increase in uterine wet weight of the stimulated horn compared to the control horn, GOF $\beta$-catenin mice did not show the same increase upon decidual stimulation (Fig. 1B), suggesting that the reduced number of implanted embryos was due, at least in part, to improper stromal cell decidualization.

\section{pSTAT3 expression is hindered in GOF $\beta$-catenin-mutant uteri during the window of uterine receptivity}

Reciprocal paracrine signaling between the stroma and epithelium is critical for rendering the uterus receptive to embryo implantation and subsequent decidualization (reviewed in: Hantak et al. 2014). Several pathways have been implicated in this crosstalk, including mitogen-activated protein kinase (MAPK)/ extracellular signal-regulated kinases $1 / 2$ (ERK1/2) (Lee et al. 2013a, Pawar et al. 2015), Janus kinase (JAK)

Published by Bioscientifica Ltd. 
A
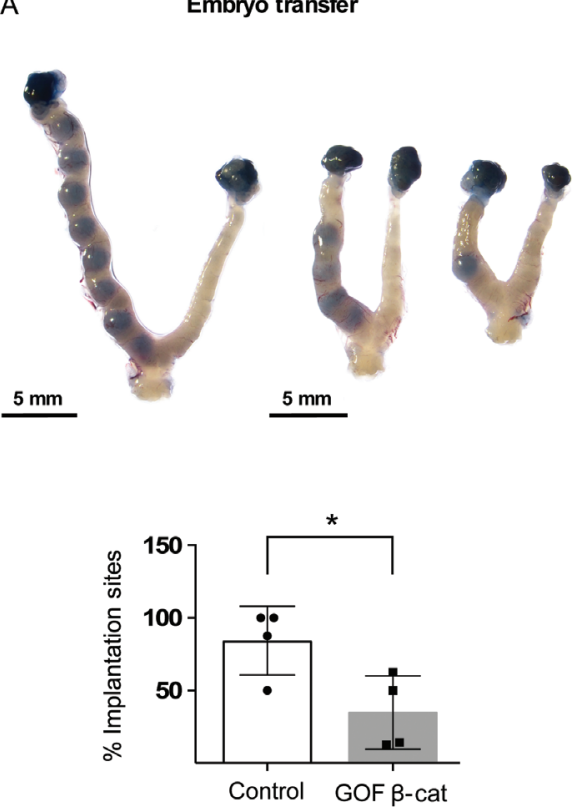

B

Artificial decidualization

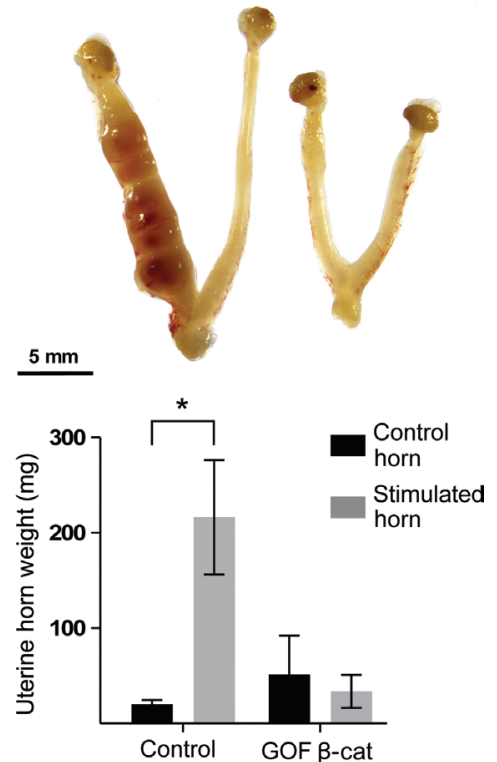

Figure 1

GOF $\beta$-catenin mice show reduced embryo implantation and decidualization. (A) Embryos (E 3.5) were surgically transferred into one uterine horn of control $(n=4)$ or GOF $\beta$-catenin $(n=4)$ mice on pseudopregnancy day 2.5 , and implantation sites were detected by Chicago blue dye. The number of implantation sites as a percentage of total embryos transferred per mouse were graphed and analyzed by unpaired $T$-test $(P=0.03)$. (B) Control $(n=3)$ and GOF $\beta$-catenin $(n=3)$ mice were mated to vasectomized male mice to induce pseudopregnancy. On day of pseudopregnancy (DOPP) 4, oil was injected into one uterine horn to induce decidualization and uteri were collected on DOPP 9. Gross anatomy images show

decidualization and was quantified by uterine wet weights. Values are the mean \pm S.E.M. compared by 2-way ANOVA $(P=0.01)$. Representative images of uteri are shown. and signal transducer and activator of transcription 3 (STAT3) (Lee et al. 2013b, Pawar et al. 2013, Sun et al. 2013) and Indian hedgehog (IHH) (Lee et al. 2006); when these pathways are perturbed, embryo implantation and decidualization can be impaired. To determine the mechanisms causing decidualization and implantation defects in GOF $\beta$-catenin mice, MAPK/ERK and JAK/STAT pathways were evaluated on DOPP 4.5 at the end of the window of uterine receptivity, just as decidualization would be initiated (Wang \& Dey 2006). The levels of phosphorylated-ERK1/2 (pERK1/2) appeared similar in control and GOF $\beta$-catenin mice (Fig. 2A). However, phosphorylated-STAT3 (pSTAT3) levels in GOF $\beta$-catenin mice were much lower compared to those in controls on DOPP 4.5 (Fig. 2B). Leukemia inhibitory factor (LIF), which is produced by the glandular epithelium (GE) in response to estrogen (Chen et al. 2000), is a wellknown activator of JAK/STAT signaling, specifically STAT3, during early pregnancy (Cheng et al. 2001, Pawar et al. 2013). Because GOF $\beta$-catenin mice have a reduced number of glands (Stewart et al. 2013), we wanted to determine if decreased estrogen and/or LIF signaling could account for the decreased pSTAT3. Serum estrogen levels were comparable (Fig. 2C), estrogen receptor alpha (ER $\alpha)$ was similarly expressed by IHC in the luminal epithelium (LE), GE and stroma (Fig. 2E, F and G) and downstream LIF (Fig. 2H) was also similar in control and GOF $\beta$-catenin mice. This would indicate that epithelial estrogen-LIF signaling is intact in GOF $\beta$-catenin mice and is probably not the cause of decreased pSTAT3.
GOF $\beta$-catenin mice have disrupted epithelial cell signaling regardless of exogenous hormone administration

Progesterone signaling is absolutely required for pregnancy and PR and STAT3 have been linked during early pregnancy (Lee et al. 2013b). Analysis of serum from GOF $\beta$-catenin mice showed reduced levels of progesterone compared to controls (Fig. 2D), which could account for the impaired implantation and decidualization and perhaps decreased pSTAT3. Therefore, exogenous progesterone and estrogen were administered to mice to mimic early pregnancy to determine whether this could rescue the loss of pSTAT3 and the decidualization defect observed in GOF $\beta$-catenin mice. LIF and pERK1/2 levels, which were unaffected in pseudopregnant (DOPP 4.5) mice with endogenous ovarian-produced hormones, were similarly unaffected in GOF $\beta$-catenin uteri with exogenous hormone treatment (Fig. 3). When hormone primed mice were given a deciduogenic stimulus (i.e. luminal scratch), GOF $\beta$-catenin mice were still incapable of mounting an appropriate decidual response (Supplementary Fig. 1A) similar to mice with endogenous ovarian-derived hormones.

The IHH signaling pathway is a well-known epithelial-stromal crosstalk pathway necessary for embryo implantation and decidualization and is regulated by progesterone (Matsumoto et al. 2002, Takamoto et al. 2002, Lee et al. 2006). Pgr, Ihh and Ptch1 mRNA levels were not very different between control and GOF $\beta$-catenin mice (Fig. 4A, B and C). Nuclear receptor subfamily 2 group F member 2 (Nr2f2; gene for

Published by Bioscientifica Ltd 
A
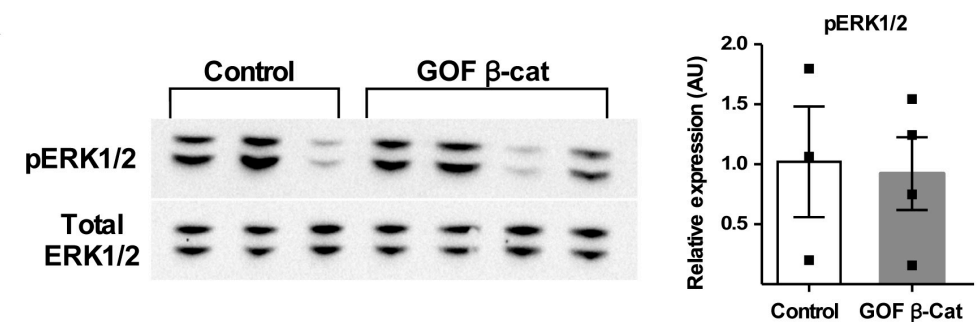

B
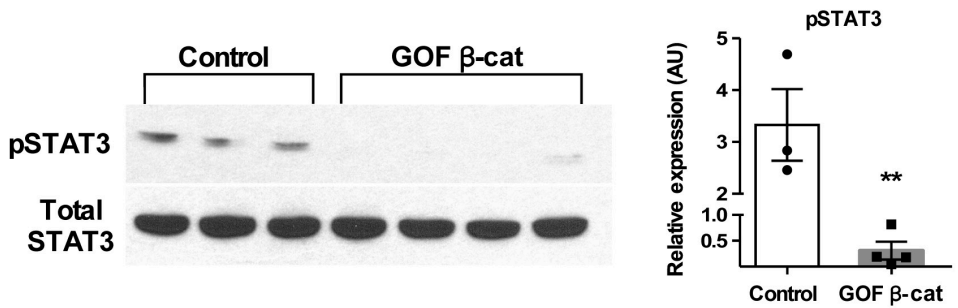

C

Serum estradiol

D Serum progesterone
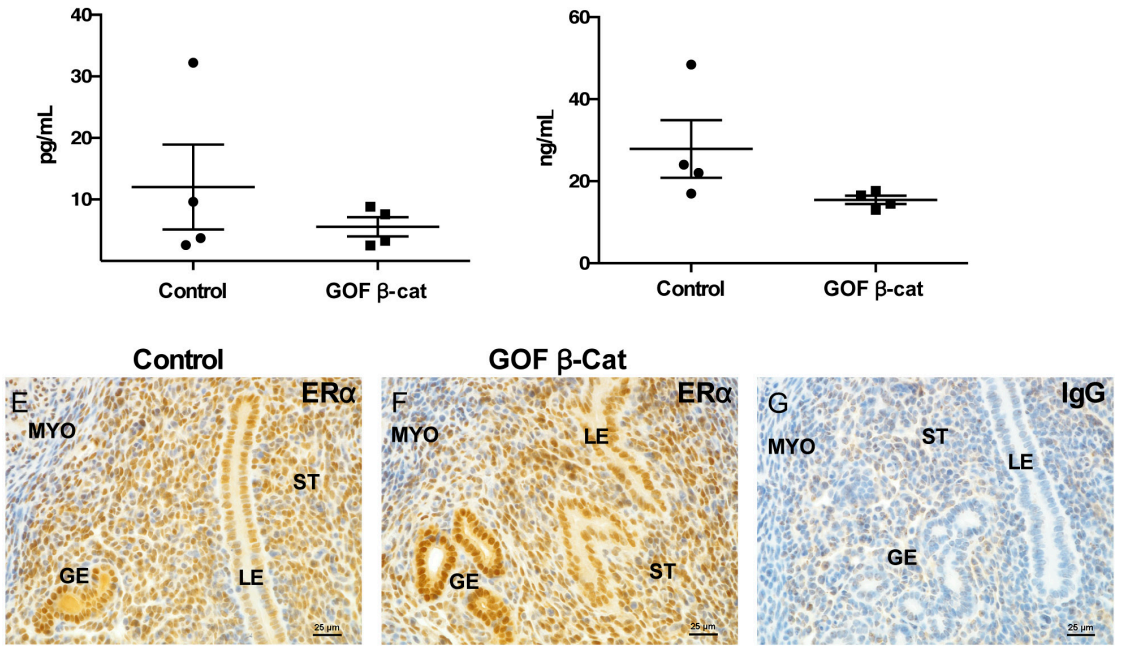

\section{Figure 2}

Expression of factors required for embryo implantation and decidualization during uterine receptivity. Pseudopregnancy was in induced in control $(n=3)$ and GOF $\beta$-catenin $(n=4)$ mice and uteri were analyzed on DOPP 4.5 for expression of factors important for implantation and decidualization. pERK1/2 (A) and pSTAT3 ( $B$; $P=0.004$ ) were semi-quantified by WB in control and GOF $\beta$-catenin mice. Serum estradiol (C) and progesterone ( $D ; P=0.057)$ levels were assessed by ELISA, and ER $\alpha(E, F$ and G) and LIF (H) were compared by IHC and WB, respectively, in control and GOF $\beta$-catenin mice. (G) IgG control for anti-ER $\alpha$ antibody. LIF expression $(\mathrm{H})$ was normalized to $\beta$-tubulin, and pERK $1 / 2$ (A) and pSTAT3 (B) were normalized to the respective total protein levels. Values are the mean \pm S.E.M. compared by $T$-test. Representative images of uterine cross-sections shown for ER $\alpha \mathrm{IHC}$. GE, glandular epithelium; LE, luminal epithelium; MYO, myometrium; ST, stroma.

COUP-TFII) and Bmp2 mRNA levels were both upregulated in GOF $\beta$-catenin mice (Fig. 4D and E). However, Wnt4, another critical player downstream of BMP2 signaling, was not significantly changed (Fig. 4F) suggesting that although Nr2f2 and Bmp2 were upregulated, there was no functional consequence.

pSTAT3 levels were decreased in pseudopregnant (DOPP 4.5) GOF $\beta$-catenin mice (Fig. 2B) that also had decreased ovarian-produced progesterone. Similarly, pSTAT3 was still downregulated in GOF $\beta$-catenin mice with exogenous progesterone and estrogen treatment (Supplementary Fig. 1B). IHC revealed that pSTAT3 was expressed predominantly in the GE and to a lesser extent in the LE and stroma of controls (Fig. 5A). In GOF $\beta$-catenin uteri, however, pSTAT3 expression was reduced in the GE and was almost entirely absent from the LE and stroma (Fig. 5B).

The consequences of decreased pSTAT3 levels in GOF $\beta$-catenin uteri were investigated by assessing downstream targets. pSTAT3 signaling in the LE has 
A

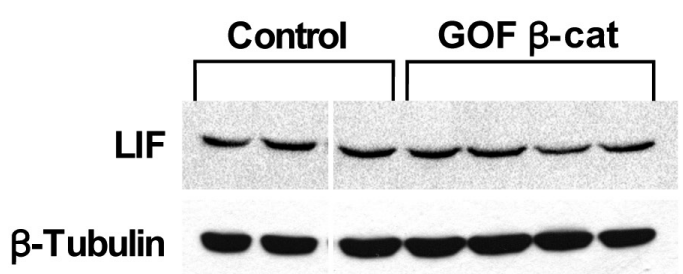

B

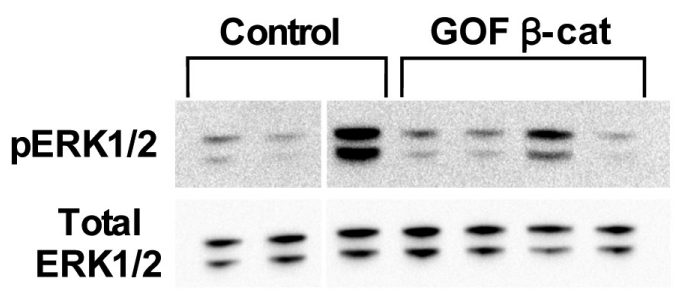

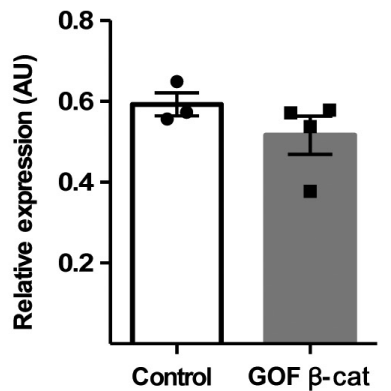

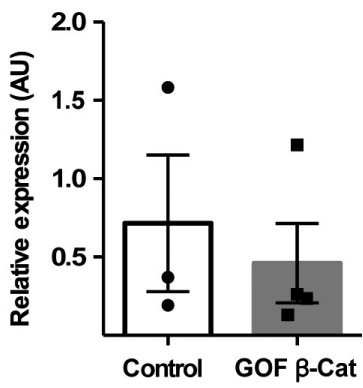

Figure 3

LIF and downstream $\mathrm{pERK} 1 / 2$ remain unchanged in GOF $\beta$-catenin mice with exogenous hormone treatment consistent with early pregnancy. Control $(n=3)$ and GOF $\beta$-catenin $(n=4)$ mice were treated with estrogen and progesterone in a manner consistent with early pregnancy as described in the 'Materials and methods' section. After treatment, protein levels of LIF (A) and PERK $1 / 2$ (B) were analyzed by WB and normalized to $\beta$-Tubulin and total ERK $1 / 2$, respectively. Values are the mean \pm S.E.M. compared by $T$-test. been shown to regulate two processes, stromal cell proliferation and epithelial cell junctional remodeling (reviewed in: Pawar et al. 2014). Additionally, Wang and coworkers generated WNT6-null mice and showed decreased stromal cell proliferation leading to a decidualization defect (Wang et al. 2013). Stromal cell proliferation was measured by Ki-67 expression and was similar between controls and GOF $\beta$-catenin mutants (Fig. 5C, D and E). Pawar and coworkers, showed that loss of epithelial STAT3 in a conditional knockout mouse model resulted in an upregulation and aberrant expression of factors associated with epithelial junctional complexes (Pawar et al. 2013). Analysis of $\alpha$-catenin (Ctnna1) and claudin 1 (Cldn1) mRNAs (Fig. 5F and G) showed that similar to the aforementioned study, both genes were upregulated, suggesting that epithelial junctional complexes were not being properly remodeled in GOF $\beta$-catenin uteri upon stimulation with early pregnancy hormones. Taken together, these data imply that GOF $\beta$-catenin in the stroma leads to reduced epithelial cell STAT3 signaling and possibly regulation of epithelial junctional complex remodeling.

\section{Stromal cells with GOF $\beta$-catenin are competent to decidualize in vitro}

Proper epithelial cell signaling is important for stromal cell decidualization. Because the epithelia in GOF $\beta$-catenin
A

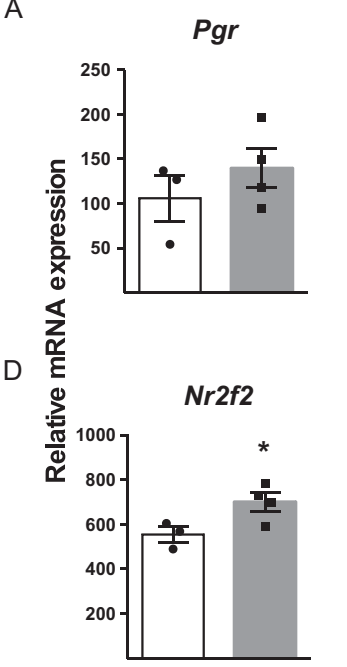

B

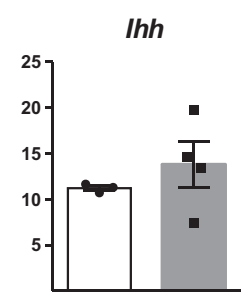

$E$

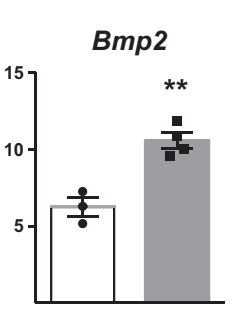

C

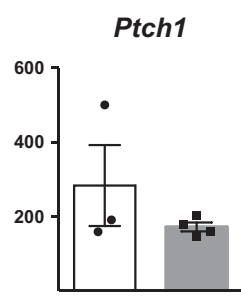

$\mathrm{F}$

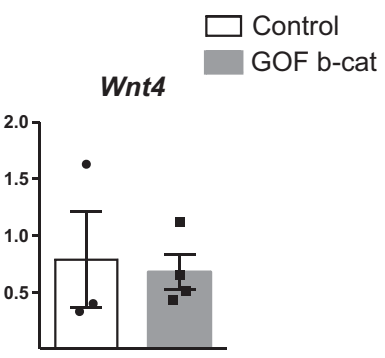

Figure 4

GOF $\beta$-catenin mice do not have impaired IHH signaling with exogenous hormone treatment. The Indian hedgehog $(\mathrm{IHH})$ signaling pathway was assessed in control $(n=3)$ and GOF $\beta$-catenin $(n=4)$ mice after administration of exogenous hormones consistent with early pregnancy. $\operatorname{Pgr}(\mathrm{A}) \operatorname{Ihh}(\mathrm{B})$ Ptch1 (C), Nr2f2 (D; $P=0.05)$ Bmp2 (E; $P=0.003$ ) and Wnt4 (F) were analyzed by qRT-PCR. Values are 2-delta $\mathrm{Ct}$ of $\mathrm{Ct}$ values that were normalized to Rp/19 and were compared by $T$-test. http://joe.endocrinology-journals.org DOI: $10.1530 / J O E-16-0502$
๑) 2017 Society for Endocrinology Printed in Great Britain 


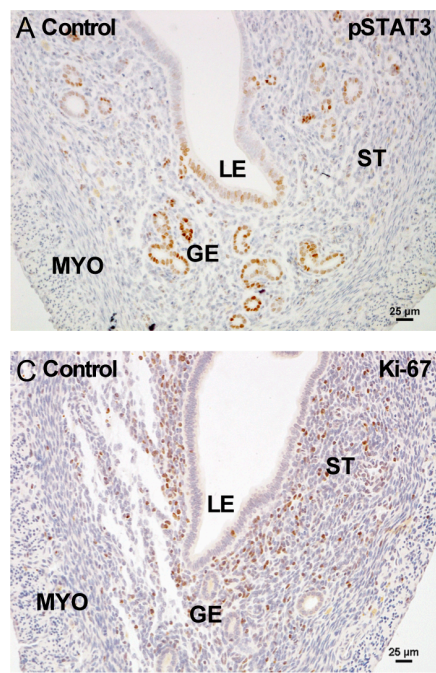

E

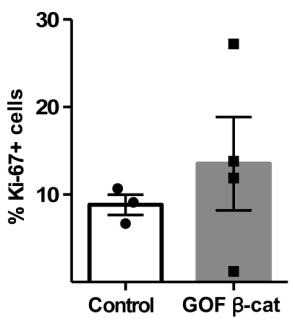

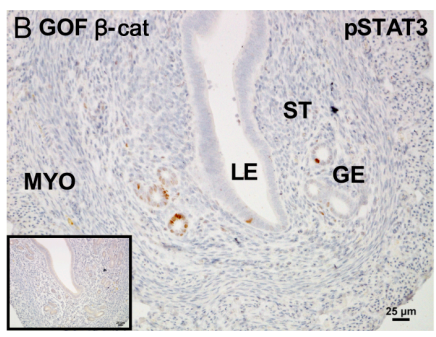

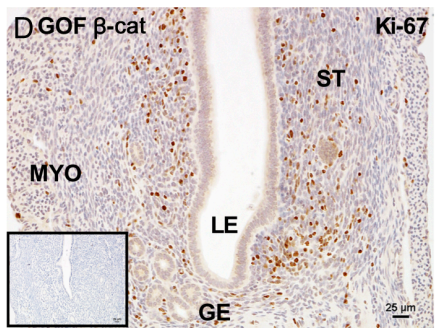

F

Ctnna1

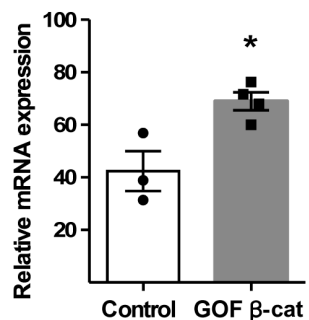

G

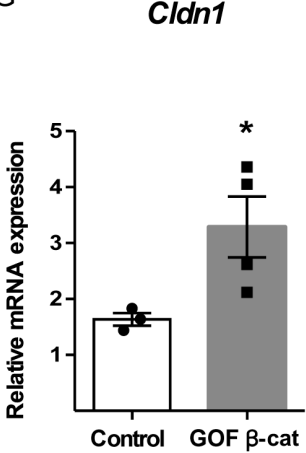

$\mathrm{H}$

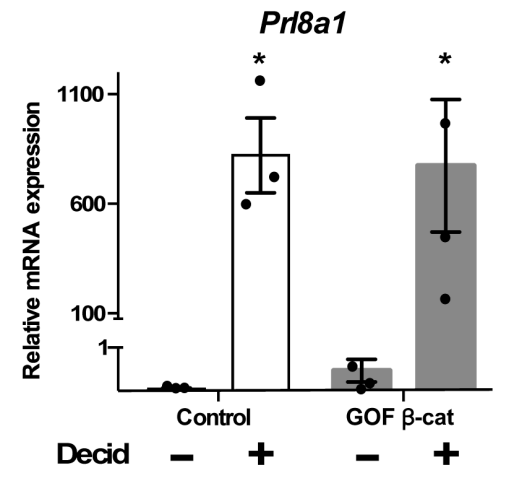

Figure 5

Altered expression of pSTAT3 and downstream junctional complex factors in GOF $\beta$-catenin mice with exogenous administration of hormones. Control $(n=3)$ and GOF $\beta$-catenin $(n=4)$ mice were treated with estrogen and progesterone to mimic early pregnancy as described in the 'Materials and methods' section. pSTAT3 ( $A$ and B) and Ki-67 (C, D and E) expression were assessed by IHC (insets: IgG control for anti-pSTAT3 or anti-Ki-67 antibodies). Expression of epithelial junctional complex factors including Ctnna1 $(F ; P=0.02)$ and $C l d n 1$ ( $G ; P=0.05)$ downstream of pSTAT3 were assessed by qRT-PCR. (H) Endometrial stromal cells were isolated from control and GOF $\beta$-catenin mice and treated with estradiol and progesterone for 8 days in vitro to induce decidualization. Pr/8a2 expression, a marker of decidualization, was determined by $q$ RT-PCR. Decid, decidualization treatment. Control $* P=0.04$, GOF $\beta$-catenin $* P=0.02$. All values are means \pm s.E.M. compared by $T$-test. Images of pSTAT3 and Ki- $67 \mathrm{IHC}$ are representative. GE, glandular epithelium; LE, luminal epithelium; MYO, myometrium; ST, stroma.

uteri were compromised, we wanted to determine whether stromal cells would be able to decidualize when epithelial cells were absent. Stromal cells were isolated from GOF $\beta$-catenin uteri and controls for in vitro decidualization with estrogen and progesterone treatment. After 8 days of treatment, mRNA expression of the decidual marker prolactin family 8 , subfamily a, member 2 (Prl8a2; Li et al. 2007) was comparably increased in control and GOF $\beta$-catenin stromal cells with treatment compared to no treatment (Fig. 5H). This indicates that GOF $\beta$-catenin stromal cells, when removed from epithelial cell influence and when provided with proper hormone stimuli, are capable of decidualizing. (c) 2017 Society for Endocrinology Printed in Great Britain

\section{Discussion}

In this study, we have shown that specific components of epithelial cell signaling were affected in uteri of mice with stromal GOF $\beta$-catenin, resulting in the implantation and decidualization defects, whereas other epithelial signaling cascades likely remained intact (Fig. 6). To determine why GOF $\beta$-catenin mice had a reduced number of implantation sites and decreased response to artificial decidualization stimulus (Fig. 1 and Supplementary Fig. 1A), expression of factors known to be involved in stromal-epithelial crosstalk were assessed during preparation of the uterus for decidualization, including 


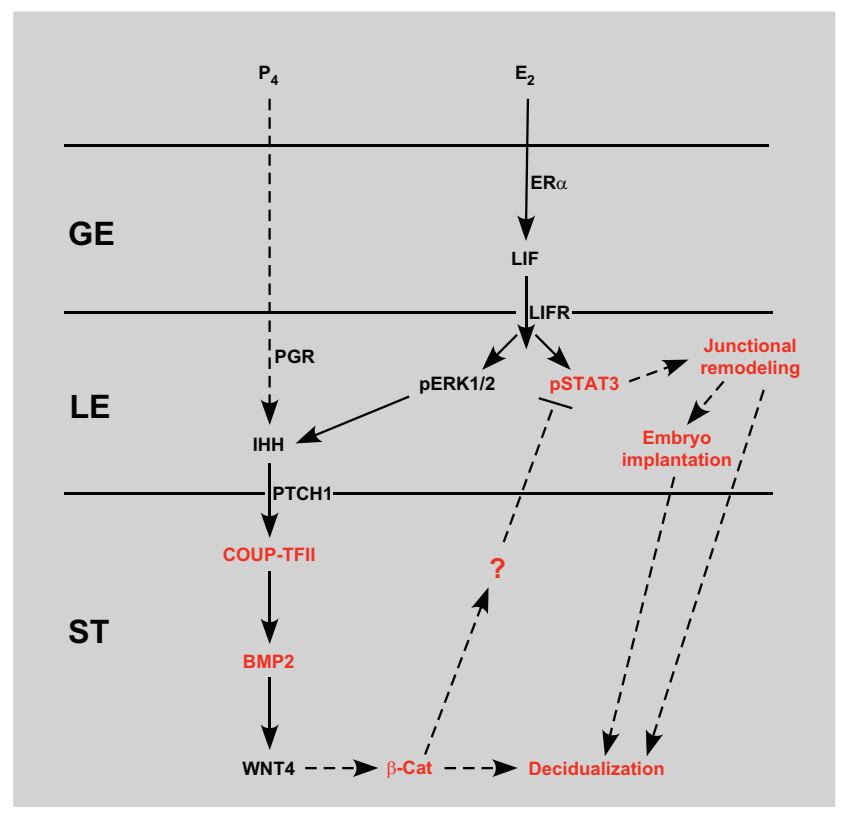

Figure 6

Schematic model of epithelial and stromal signaling pathways affected in GOF $\beta$-catenin mice during implantation and decidualization. Estradiol signals through its receptor, $E R \alpha$, in the glandular epithelium (GE) to induce LIF expression. LIF signals through its receptor, LIFR, in the luminal epithelium (LE) to activate ERK and JAK/STAT signaling. ERK signaling in concert with progesterone signaling through PR induces the expression of IHH in the LE. IHH signals through PTCH1 in the stroma (ST) to upregulate the COUP-TFII-BMP2-WNT4 pathway. WNT4 may then signal through $\beta$-catenin to regulate decidualization. GOF $\beta$-catenin perturbs this pathway presumably by regulating the expression of an unknown factor that signals to the LE to affect phosphorylation of STAT3 leading to altered expression of junctional remodeling factors and impaired embryo implantation and decidualization. Black lettering, un-altered expression; red lettering, altered expression/function.

the MAPK/ERK, JAK/STAT and IHH pathways, all of which are required for both implantation and decidualization. Estrogen signaling seemed to be intact in the epithelium as seen by comparable levels of serum estrogen, ER $\alpha$ and/ or LIF expression (Figs 2 and 3). Stewart and coworkers showed, using the same mouse model used in this study, that GOF $\beta$-catenin uteri have significantly fewer glands (Stewart et al. 2013), which we have also observed. It is interesting that although GOF $\beta$-catenin uteri have fewer glands they are still able to produce sufficient amounts of LIF, which is critical for uterine receptivity and embryo implantation (Stewart et al. 1992). Furthermore, ERK1/2 activation (e.g. pERK1/2), which was shown to be downstream of LIF signaling in the LE (Pawar et al. 2015), was not altered in GOF $\beta$-catenin uteri. The IHH signaling pathway that is initiated in the LE and concludes in the stroma also appeared to be predominantly intact in hormone-treated mice, with the exception of $\mathrm{Nr} 2 \mathrm{f2}$ (gene for COUP-TFII) and Bmp2, both downstream stromal factors, which were upregulated (Fig. 4). $\beta$-Catenin has been shown to activate BMP2 expression in osteoblasts (Zhang et al. 2013) and COUP-TFII in adipocytes (Okamura et al. 2009); therefore, it is not surprising that we found increased levels of Bmp2 and Nr2f2 in GOF $\beta$-catenin uteri. It is unclear at this time whether increased $B m p 2$ and $N r 2 f 2$ have any negative impact on decidualization. However, because downstream Wnt4 was unchanged, it is unlikely.

Of note, supplementation of exogenous progesterone did not rescue the reduced levels of pSTAT3 or the impaired decidual response seen in pseudopregnant GOF $\beta$-catenin mice that had decreased endogenous progesterone (Supplementary Fig. 1). Lee and coworkers reported on the direct interaction between STAT3 and PR and showed that conditional loss of STAT3 using Pgr-Cre resulted in reduced stromal PR, impaired progesterone signaling and defective implantation and decidualization (Lee et al. 2013b). The authors concluded that STAT3 was required for proper PR signaling. Because of the decrease in pSTAT3 observed in GOF $\beta$-catenin mice, we had anticipated seeing a similar effect on PR signaling. However, progesterone-regulated IHH signaling appeared to be intact. Differences between the two studies such as the specific PR pathways that were evaluated, different Cre models used (Pgr-Cre vs Amhr2Cre) and that STAT3 was not specifically deleted in our study, could account for the varied results. Additional studies are needed to further understand the possible PR-STAT3 interactions in the context of GOF $\beta$-catenin.

Pawar and coworkers showed that conditional knockout of STAT3 in endometrial epithelium resulted in decreased stromal cell proliferation and an increase in several factors involved in epithelial junctional complexes (Pawar et al. 2013). Likewise, decreased pSTAT3 was correlated with increased expression of Ctnna1 and Cldn1 mRNA (junctional complex factors) (Fig. 5); however, decreased stromal cell proliferation was not observed. This discrepancy is likely due to timing; we assessed proliferation prior to decidualization, whereas Parwar and coworkers evaluated proliferation $20 \mathrm{~h}$ after decidual stimulation. Additionally, because $\beta$-catenin itself is a stimulator of proliferation, it is possible that GOF $\beta$-catenin was capable of stimulating stromal cell proliferation even in the absence of pSTAT3-stimulated proliferation. Junctional complex proteins, such as occludins and claudins, have a tightly regulated spatiotemporal pattern of expression during implantation and decidualization, and remodeling of these complexes may 
lead to a loss of epithelial apical-basal polarity allowing for blastocyst attachment to the LE (Thie et al. 1996, Wang et al. 2004, Pawar et al. 2013, Schumann et al. 2015). It is also possible that dismantling of junctional complexes allows for breakdown of the LE at the implantation site to allow for blastocyst invasion. It is tempting to speculate that in GOF $\beta$-catenin mice, decreased epithelial pSTAT3 leads to increased expression of junctional complex proteins, followed by impaired junctional remodeling and LE breakdown, which could lead to decreased embryo implantation and stromal decidualization. Our data showing that purified stromal cells from GOF $\beta$-catenin uteri, when removed from epithelial cell influence and provided with the appropriate hormone stimuli, are able to decidualize in vitro, support this possibility.

It is notable that although the GOF $\beta$-catenin protein is only expressed in the stroma and myometrium and not in the epithelium, the epithelium appears to be most affected in our study. However, it is well known that the stroma dictates epithelial cell fate during development (Cunha 1976, Kurita et al. 2001) and has a large influence on epithelial cell function in the adult (Cooke et al. 1997, Kurita et al. 2000, Winuthayanon et al. 2010). Further, Stewart and coworkers used the same mouse model of GOF $\beta$-catenin in the stroma and myometrium and reported on the effects exhibited on the epithelium (Stewart et al. 2013). They showed that epithelial-tomesenchymal transition occurred in the LE prior to birth and that adenogenesis was impaired postnatally resulting in fewer endometrial glands in the adult. Our study combined with the aforementioned study provides compelling evidence for the role of stromal $\beta$-catenin in regulating epithelial development and function. What is unclear at this point is what factors are produced in the stroma in response to $\beta$-catenin that then signal to and affect epithelial cell function (Fig. 6). It is also unknown whether the dysfunction in epithelial cells triggered by stromal GOF $\beta$-catenin, in the current study, resulted from perturbed epithelial development or is a direct result of GOF $\beta$-catenin in the adult or both. These questions open up exciting avenues for future studies. What is apparent is that $\beta$-catenin activity must be tightly regulated during uterine development and pregnancy, as both loss of function and GOF result in fertility defects in adult mice.

In summary, expression of GOF $\beta$-catenin in the MDM-derived stroma and myometrium resulted in implantation and decidualization defects. We propose that these defects were due to improper epithelial cell function as a result of decreased pSTAT3 and increased expression of Ctnna1 and Cldn1, components of junctional complexes. Isolated stromal cells from GOF $\beta$-catenin mice decidualized in vitro further supporting the idea that compromised epithelial cell function and possibly stromalepithelial crosstalk were responsible for impaired embryo implantation and stromal cell decidualization in vivo.

\section{Supplementary data}

This is linked to the online version of the paper at http://dx.doi.org/10.1530/ JOE-16-0502.

Declaration of interest

The authors declare that there is no conflict of interest that could be perceived as prejudicing the impartiality of the research reported.

\section{Funding}

This research was funded by NIH HD072489. ALP is supported by NIH F32HD081856.

Author contribution statement

A L P and J M T designed and conducted or oversaw all of the experiments and wrote the manuscript. J P and S Y T conducted key experiments documented in the manuscript.

\section{Acknowledgements}

The authors thank Audra Guikman for conducting the embryo transfer experiments and The University of Virginia Center for Research in Reproduction Ligand Assay and Analysis Core (supported by the Eunice Kennedy Shriver NICHD/NIH (NCTRI) Grant P50-HD28934) for serum hormone assays.

\section{References}

Arango NA, Szotek PP, Manganaro TF, Oliva E, Donahoe PK \& Teixeira J 2005 Conditional deletion of beta-catenin in the mesenchyme of the developing mouse uterus results in a switch to adipogenesis in the myometrium. Developmental Biology 288 276-283. (doi:10.1016/j. ydbio.2005.09.045)

Boerboom D, Paquet M, Hsieh M, Liu J, Jamin SP, Behringer RR, Sirois J, Taketo MM \& Richards JS 2005 Misregulated wnt/beta-catenin signaling leads to ovarian granulosa cell tumor development. Cancer Research 65 9206-9215. (doi:10.1158/0008-5472.CAN-05-1024)

Chen JR, Cheng JG, Shatzer T, Sewell L, Hernandez L \& Stewart CL 2000 Leukemia inhibitory factor can substitute for nidatory estrogen and is essential to inducing a receptive uterus for implantation but is not essential for subsequent embryogenesis. Endocrinology 141 4365-4372. (doi:10.1210/endo.141.1.7237)

Cheng JG, Chen JR, Hernandez L, Alvord WG \& Stewart CL 2001 Dual control of lif expression and lif receptor function regulate stat3 activation at the onset of uterine receptivity and embryo implantation. PNAS 98 8680-8685. (doi:10.1073/pnas.151180898)

Published by Bioscientifica Ltd. 
Cooke PS, Buchanan DL, Young P, Setiawan T, Brody J, Korach KS Taylor J, Lubahn DB \& Cunha GR 1997 Stromal estrogen receptors mediate mitogenic effects of estradiol on uterine epithelium. PNAS 94 6535-6540. (doi:10.1073/pnas.94.12.6535)

Cunha GR 1976 Stromal induction and specification of morphogenesis and cytodifferentiation of the epithelia of the mullerian ducts and urogenital sinus during development of the uterus and vagina in mice. Journal of Experimental Zoology 196 361-370. (doi:10.1002/ jez.1401960310)

Deutscher E \& Hung-Chang Yao H 2007 Essential roles of mesenchyme-derived beta-catenin in mouse mullerian duct morphogenesis. Developmental Biology 307 227-236. (doi:10.1016/j. ydbio.2007.04.036)

Dunlap KA, Filant J, Hayashi K, Rucker EB 3rd, Song G, Deng JM, Behringer RR, Demayo FJ, Lydon J, Jeong JW, et al. 2011 Postnatal deletion of wnt7a inhibits uterine gland morphogenesis and compromises adult fertility in mice. Biology of Reproduction $\mathbf{8 5}$ 386-396. (doi:10.1095/biolreprod.111.091769)

Franco HL, Dai D, Lee KY, Rubel CA, Roop D, Boerboom D, Jeong JW, Lydon JP, Bagchi IC, Bagchi MK, et al. 2011 Wnt4 is a key regulator of normal postnatal uterine development and progesterone signaling during embryo implantation and decidualization in the mouse. FASEB Journal 25 1176-1187. (doi:10.1096/fj.10-175349)

Hantak AM, Bagchi IC \& Bagchi MK 2014 Role of uterine stromalepithelial crosstalk in embryo implantation. International Journal of Developmental Biology 58 139-146. (doi:10.1387/ijdb.130348mb)

Harada N, Tamai Y, Ishikawa T, Sauer B, Takaku K, Oshima M \& Taketo MM 1999 Intestinal polyposis in mice with a dominant stable mutation of the beta-catenin gene. EMBO Journal 18 5931-5942. (doi:10.1093/emboj/18.21.5931)

Jamin SP, Arango NA, Mishina Y, Hanks MC \& Behringer RR 2002 Requirement of Bmpr1a for Mullerian duct regression during male sexual development. Nature Genetics 32 408-410. (doi:10.1038/ ng1003)

Kurihara I, Lee DK, Petit FG, Jeong J, Lee K, Lydon JP, Demayo FJ, Tsai MJ \& Tsai SY 2007 Coup-tfii mediates progesterone regulation of uterine implantation by controlling er activity. PLoS Genetics 3 e102. (doi:10.1371/journal.pgen.0030102)

Kurita T, Lee KJ, Cooke PS, Taylor JA, Lubahn DB \& Cunha GR 2000 Paracrine regulation of epithelial progesterone receptor by estradiol in the mouse female reproductive tract. Biology of Reproduction $\mathbf{6 2}$ 821-830. (doi:10.1093/biolreprod/62.4.821)

Kurita T, Cooke PS \& Cunha GR 2001 Epithelial-stromal tissue interaction in paramesonephric (mullerian) epithelial differentiation. Developmental Biology 240 194-211. (doi:10.1006/dbio.2001.0458)

Lee K, Jeong J, Kwak I, Yu CT, Lanske B, Soegiarto DW, Toftgard R, Tsai MJ, Tsai S, Lydon JP, et al. 2006 Indian hedgehog is a major mediator of progesterone signaling in the mouse uterus. Nature Genetics $\mathbf{3 8}$ 1204-1209. (doi:10.1038/ng1874)

Lee KY, Jeong JW, Wang J, Ma L, Martin JF, Tsai SY, Lydon JP \& Demayo FJ 2007 Bmp2 is critical for the murine uterine decidual response. Molecular and Cellular Biology 27 5468-5478. (doi:10.1128/ MCB.00342-07)

Lee CH, Kim TH, Lee JH, Oh SJ, Yoo JY, Kwon HS, Kim YI, Ferguson SD, Ahn JY, Ku BJ, et al. 2013a Extracellular signal-regulated kinase 1/2 signaling pathway is required for endometrial decidualization in mice and human. PLOS ONE 8 e75282. (doi:10.1371/journal.pone.0075282)

Lee JH, Kim TH, Oh SJ, Yoo JY, Akira S, Ku BJ, Lydon JP \& Jeong JW $2013 b$ Signal transducer and activator of transcription-3 (stat3) plays a critical role in implantation via progesterone receptor in uterus. FASEB Journal 27 2553-2563. (doi:10.1096/fj.12-225664)

Li Q, Kannan A, Wang W, Demayo FJ, Taylor RN, Bagchi MK \& Bagchi IC 2007 Bone morphogenetic protein 2 functions via a conserved signaling pathway involving wnt4 to regulate uterine decidualization in the mouse and the human. Journal of Biological Chemistry $\mathbf{2 8 2}$ 31725-31732. (doi:10.1074/jbc.M704723200)
Li Q, Kannan A, Das A, Demayo FJ, Hornsby PJ, Young SL, Taylor RN, Bagchi MK \& Bagchi IC 2013 Wnt4 acts downstream of bmp2 and functions via beta-catenin signaling pathway to regulate human endometrial stromal cell differentiation. Endocrinology 154 446-457. (doi:10.1210/en.2012-1585)

Matsumoto H, Zhao X, Das SK, Hogan BL \& Dey SK 2002 Indian hedgehog as a progesterone-responsive factor mediating epithelialmesenchymal interactions in the mouse uterus. Developmental Biology 245 280-290. (doi:10.1006/dbio.2002.0645)

Mericskay M, Kitajewski J \& Sassoon D 2004 Wnt5a is required for proper epithelial-mesenchymal interactions in the uterus. Development 131 2061-2072. (doi:10.1242/dev.01090)

Mohamed OA, Jonnaert M, Labelle-Dumais C, Kuroda K, Clarke HJ \& Dufort D 2005 Uterine wnt/beta-catenin signaling is required for implantation. PNAS 102 8579-8584. (doi:10.1073/pnas.0500612102)

Okamura M, Kudo H, Wakabayashi K, Tanaka T, Nonaka A, Uchida A, Tsutsumi S, Sakakibara I, Naito M, Osborne TF, et al. 2009 Coup-tfii acts downstream of wnt/beta-catenin signal to silence ppargamma gene expression and repress adipogenesis. PNAS 106 5819-5824. (doi:10.1073/pnas.0901676106)

Parr BA \& McMahon AP 1998 Sexually dimorphic development of the mammalian reproductive tract requires Wnt-7a. Nature 395 707-710. (doi:10.1038/27221)

Pawar S, Starosvetsky E, Orvis GD, Behringer RR, Bagchi IC \& Bagchi MK 2013 Stat3 regulates uterine epithelial remodeling and epithelialstromal crosstalk during implantation. Molecular Endocrinology 27 1996-2012. (doi:10.1210/me.2013-1206)

Pawar S, Hantak AM, Bagchi IC \& Bagchi MK 2014 Minireview: steroidregulated paracrine mechanisms controlling implantation. Molecular Endocrinology 28 1408-1422. (doi:10.1210/me.2014-1074)

Pawar S, Laws MJ, Bagchi IC \& Bagchi MK 2015 Uterine epithelial estrogen receptor-alpha controls decidualization via a paracrine mechanism. Molecular Endocrinology 29 1362-1374. (doi:10.1210/ me.2015-1142)

Prunskaite-Hyyrylainen R, Skovorodkin I, Xu Q, Miinalainen I, Shan J \& Vainio SJ 2016 Wnt4 coordinates directional cell migration and extension of the mullerian duct essential for ontogenesis of the female reproductive tract. Human Molecular Genetics 25 1059-1073. (doi:10.1093/hmg/ddv621)

Ramathal CY, Bagchi IC, Taylor RN \& Bagchi MK 2010 Endometrial decidualization: of mice and men. Seminars in Reproductive Medicine $\mathbf{2 8}$ 17-26. (doi:10.1055/s-0029-1242989)

Rubel CA, Lanz RB, Kommagani R, Franco HL, Lydon JP \& Demayo FJ 2012 Research resource: genome-wide profiling of progesterone receptor binding in the mouse uterus. Molecular Endocrinology 26 1428-1442. (doi:10.1210/me.2011-1355)

Schumann S, Buck VU, Classen-Linke I, Wennemuth G \& Grummer R 2015 Claudin-3, claudin-7, and claudin-10 show different distribution patterns during decidualization and trophoblast invasion in mouse and human. Histochemistry and Cell Biology 144 571-585. (doi:10.1007/s00418-015-1361-z)

Stewart CL, Kaspar P, Brunet LJ, Bhatt H, Gadi I, Kontgen F \& Abbondanzo SJ 1992 Blastocyst implantation depends on maternal expression of leukaemia inhibitory factor. Nature 359 76-79. (doi:10.1038/359076a0)

Stewart CA, Wang Y, Bonilla-Claudio M, Martin JF, Gonzalez G, Taketo MM \& Behringer RR 2013 Ctnnb1 in mesenchyme regulates epithelial cell differentiation during mullerian duct and postnatal uterine development. Molecular Endocrinology 27 1442-1454. (doi:10.1210/ me.2012-1126)

Sun X, Bartos A, Whitsett JA \& Dey SK 2013 Uterine deletion of gp130 or stat3 shows implantation failure with increased estrogenic responses. Molecular Endocrinology 27 1492-1501. (doi:10.1210/me.2013-1086)

Takamoto N, Zhao B, Tsai SY \& Demayo FJ 2002 Identification of indian hedgehog as a progesterone-responsive gene in the murine uterus. Molecular Endocrinology 16 2338-2348. (doi:10.1210/me.2001-0154) 
Tanwar PS, Lee HJ, Zhang L, Zukerberg LR, Taketo MM, Rueda BR \& Teixeira JM 2009 Constitutive activation of beta-catenin in uterine stroma and smooth muscle leads to the development of mesenchymal tumors in mice. Biology of Reproduction 81 545-552. (doi:10.1095/ biolreprod.108.075648)

Thie M, Fuchs P \& Denker HW 1996 Epithelial cell polarity and embryo implantation in mammals. International Journal of Developmental Biology 40 389-393.

Vainio S, Heikkila M, Kispert A, Chin N \& McMahon AP 1999 Female development in mammals is regulated by Wnt-4 signalling. Nature 397 405-409. (doi:10.1038/17068)

Valenta T, Hausmann G \& Basler K 2012 The many faces and functions of beta-catenin. EMBO Journal 31 2714-2736. (doi:10.1038/ emboj.2012.150)

Wang H \& Dey SK 2006 Roadmap to embryo implantation: clues from mouse models. Nature Reviews Genetics 7 185-199. (doi:10.1038/ nrg1808)

Wang X, Matsumoto H, Zhao X, Das SK \& Paria BC 2004 Embryonic signals direct the formation of tight junctional permeability barrier in the decidualizing stroma during embryo implantation. Journal of Cell Science 117 53-62. (doi:10.1242/jcs.00826)

Wang Q, Lu J, Zhang S, Wang S, Wang W, Wang B, Wang F, Chen Q, Duan E, Leitges M, et al. 2013 Wnt6 is essential for stromal cell proliferation during decidualization in mice. Biology of Reproduction 88 5. (doi:10.1095/biolreprod.112.104687)

Winuthayanon W, Hewitt SC, Orvis GD, Behringer RR \& Korach KS 2010 Uterine epithelial estrogen receptor alpha is dispensable for proliferation but essential for complete biological and biochemical responses. PNAS 107 19272-19277. (doi:10.1073/ pnas.1013226107)

Zhang L, Patterson AL, Zhang L, Teixeira JM \& Pru JK 2012 Endometrial stromal beta-catenin is required for steroid-dependent mesenchymalepithelial cross talk and decidualization. Reproductive Biology and Endocrinology 10 75. (doi:10.1186/1477-7827-10-75)

Zhang R, Oyajobi BO, Harris SE, Chen D, Tsao C, Deng HW \& Zhao M 2013 Wnt/beta-catenin signaling activates bone morphogenetic protein 2 expression in osteoblasts. Bone 52 145-156. (doi:10.1016/j. bone.2012.09.029)

Received in final form 27 January 2017

Accepted 9 February 2017

Accepted Preprint published online 9 February 2017 (c) 2017 Society for Endocrinology Printed in Great Britain
Published by Bioscientifica Ltd. 